\title{
Collective behavior of coupled mesoscopic cylinders
}

\author{
J. Dajka ${ }^{*}, 1$ J. Luczka ${ }^{1}$, M. Szopa ${ }^{1}$, and P. Hänggi ${ }^{2}$ \\ ${ }^{1}$ Institute of Physics, University of Silesia, 40-007 Katowice, Poland \\ ${ }^{2}$ Institute of Physics, University of Augsburg, Universitätsstr.1, 86135 Augsburg, Germany
}

Received 3 September 2004, revised 5 October 2004, accepted 5 October 2004

Published online 20 January 2005

PACS 05.10.Gg, 64.60.Cn, 73.23.-b

We address the objective of the generation of finite magnetic flux out of unbiased thermal current fluctuations in a collection of identical mesoscopic cylinders which are coupled via mutual inductances. The influence of thermal Nyquist fluctuations are described in terms of a set of Langevin equations or a corresponding Fokker-Planck equation, respectively. In the limit of infinitely many constituents, the steadystate of the system is determined by an effective, nonlinear Fokker-Planck equation. The system exhibits in this thermodynamic limit a second-order phase transition: the average flux through each cylinder changes continuously from zero to non-zero value and the phase diagram depicts a critical line.

(C) 2005 WILEY-VCH Verlag GmbH \& Co. KGaA, Weinheim

\section{Introduction}

Due to their intermediate size between the macro- and micro-world mesoscopic systems exhibit a rich variety of phenomena that are both of quantum and classical origin [1]. Herein, we elaborate on the phenomenon of finite flux generation out of current fluctuations of zero average in a system of interacting mesoscopic cylinders. More specifically, we consider a linear chain of coaxial mesoscopic cylinders that are coupled by mutual inductances. Recently, the idea of a flux phase state has been proposed in the context with both superconductivity and the topic of non-transport ground state currents in a mesoscopic normal metal samples [1,2] of multiply connected mesoscopic cylinders, rings or even carbon nanotubes [3]. The flux state [2] is characterized by a non-vanishing selfsustaining current in the system. Because of thermal equilibrium fluctuations (Nyquist noise) these selfsustaining currents in mesoscopic cylinders are - although long lasting - only metastable states of the system (in the sense that the averaged current in an equilibrium state vanishes). Due to the time-reversal symmetry, the mean flux in a finite system is always zero. The situation changes drastically when co-operativity comes into play as is the case with noise induced phase transitions within the "thermodynamic limit" of an infinite number of interacting cylinders. The model of an infinite chain of mesoscopic cylinders formed of long wires made of singlewall carbon nanotubes presents an idealized archetype.

In mesoscopic systems of the cylindrical symmetry persistent currents can occur [5] due to the quantum size effect resulting in the spatial quantisation of energy levels. Those currents emerge as a result of the phase coherence among electrons, the so-called coherent electrons. In the ground state, at temperature $T=0$, the only electrons present in the system are coherent ones possessing a non-dissipative flow. At non-zero temperature, $T>0$, a part of those electrons become "normal" and their behavior is dissipative resulting in a decrease of the amplitude of the persistent current. This feature has been confirmed experimentally in mesoscopic rings connected to a current source [6].

\footnotetext{
Corresponding author: e-mail: dajka@server.phys.us.edu.pl, Phone: +48 323591173
} 


\section{Model}

To start, let us assume that the real 3-dimensional mesoscopic cylinder is modeled as a collection of $N_{c}$ one dimensional rings (current channels) stacked along a certain axis. The geometry of cylinder is chosen in order to obtain a thin-wall system with sufficiently large current-amplitude. The coherent current is a sum of contributions of single channels which can produce currents being either paramagnetic for an even number, or diamagnetic for an odd number $N_{e}$ of coherent electrons. For simplicity, we assume that the probability of finding a channel with an odd number of coherent electrons equals the probability of finding a channel with an even number of coherent electrons. Thermal, dissipative conduction causes various sources of random fluctuations [7]. There are so-called universal conductance fluctuations that arise from the random quantum interference between many electron paths which contribute to the conductance in the diffusive regime. These fluctuations decay algebraically with temperature and can be neglected at higher temperatures [8]. There is also a part of the current noise which is called shot noise [7], the spectral density of which is proportional to mean current. This noise can be reduced by increasing the size of rings [1]. Thermal motion of charge carriers in any conductor is a source of random fluctuations of current which is called Nyquist noise [7]. This thermal equilibrium noise is universal and exists in any conductor. Moreover, this noise increases with temperature. In the following we limit our considerations to conditions of relatively high temperature and sufficiently large circumference $l_{x}$ of the cylinder when the only significant source of randomness is Nyquist noise [4].

For a system of $N$ identical mesoscopic cylinders, fluxes and currents in the cylinders (in the absence of an externally applied flux) are coupled according to the formula $[9,10]$

$$
\phi_{i}=\sum_{k=1}^{N} \mathcal{M}_{i k} I_{k},
$$

where $\phi_{i}$ and $I_{i}$ are flux and current in the $i$-th cylinder, respectively. The coupling coefficients $\mathcal{M}_{i k}=\mathcal{M}_{k i}$ (forming the matrix $\mathcal{M}$ ) denote the mutual inductances for $i \neq k$ and identical selfinductances $\mathcal{L}=\mathcal{M}_{i i}$ for $i=k$ [10]. The current in the $k$-th cylinder consists of two contributions [11]:

$$
I_{k}=I_{k}^{\mathrm{nor}}+I_{k}^{\mathrm{coh}}
$$

with the Ohmic (dissipative) current $I_{k}^{\text {nor }}=I_{\text {nor }}\left(\phi_{k}\right)$, according to the Ohm's law and Lenz's rule assuming the form

$$
I_{\text {nor }}\left(\phi_{k}\right)=-\frac{1}{R} \frac{\mathrm{d} \phi_{k}}{\mathrm{~d} t}+\sqrt{\frac{2 k_{\mathrm{B}} T}{R}} \Gamma_{k}(t) .
$$

Herein, $R$ denotes a resistance of a single cylinder [12], $k_{\mathrm{B}}$ is the Boltzmann constant, and $\Gamma_{k}(t)$ describes fluctuations of the current; i.e. thermal Nyquist noise modeled by Gaussian white noise of zero average $\left\langle\Gamma_{k}(t)\right\rangle=0$ and the Dirac $\delta$-correlations $\left\langle\Gamma_{k}(t) \Gamma_{i}(s)\right\rangle=\delta_{k i} \delta(t-s)$. Its intensity $D_{0}=\sqrt{2 k_{\mathrm{B}} T / R}$ is chosen to obey the fluctuation-dissipation theorem. The coherent electrons $I_{k}^{\mathrm{coh}}=I_{\text {coh }}\left(\phi_{k}, T\right)$ obey [4]

$$
I_{\text {coh }}\left(\phi_{k}, T\right)=\frac{N_{c} I_{0}}{2}\left(g\left(\phi_{k} / \phi_{0}, T\right)+g\left(\phi_{k} / \phi_{0}+\frac{1}{2}, T\right)\right),
$$

where the flux quantum $\phi_{0}:=h / e, I_{0}=h e N_{e} /\left(2 l_{x}^{2} m_{e}\right), N_{e}$ denotes the number of coherent electrons (each of mass $\left.m_{e}\right)$ in a single current channel and $g(x, T)=\sum_{n=1}^{\infty} A_{n}(T) \sin (2 n \pi x)$ stands for the current in a channel with an even number of coherent electrons. The amplitude equals

$$
A_{n}(T)=\frac{4 T}{\pi T^{*}} \frac{\exp \left(-n T / T^{*}\right)}{1-\exp \left(-2 n T / T^{*}\right)} \cos \left(n k_{F} l_{x}\right) .
$$


The characteristic temperature $T^{*}$ is defined by the relation $k_{\mathrm{B}} T^{*}=\Delta_{F} / 2 \pi^{2}$, where $\Delta_{F}$ is the energy gap at the Fermi surface and $k_{F}$ denotes the Fermi momentum. Inserting (2)-(4) into (1) yields

$$
\frac{1}{R} \sum_{k=1}^{N} M_{i k} \frac{\mathrm{d} \phi_{k}}{\mathrm{~d} t}=-\phi_{i}+\sum_{k=1}^{N} \mathcal{M}_{i k} I_{\mathrm{coh}}\left(\phi_{k}, T\right)+\sqrt{\frac{2 k_{\mathrm{B}} T}{R}} \sum_{k=1}^{N} \mathcal{M}_{i k} \Gamma_{k}(t)
$$

where $i=1 \ldots N$. This set of equations can be inverted to read

$$
\frac{1}{R} \frac{\mathrm{d} \phi_{i}}{\mathrm{~d} t}=I_{\mathrm{coh}}\left(\phi_{i}, T\right)-\sum_{j=1}^{N}\left(\mathcal{M}^{-1}\right)_{i j} \phi_{j}+\sqrt{\frac{2 k_{B} T}{R}} \Gamma_{i}(t) .
$$

Introducing the dimensionless flux $x_{i}=\phi_{i} / \phi_{0}$ and time $s=t / \tau_{0}$, where $\tau_{0}=\mathcal{L} / R$, from (7) we obtain (the dot indicates the derivative with respect to $x_{i}$ )

$$
\dot{x}_{i}=-V^{\prime}\left(x_{i}, T\right)-\sum_{j \neq i)}^{N} \mathcal{L}\left(\mathcal{M}^{-1}\right)_{i j} x_{j}+\sqrt{2 D} \tilde{\Gamma}_{i}(s),
$$

where

$$
V\left(x_{i}, T\right)=\frac{1}{2} a_{i} x_{i}^{2}-i_{0}^{x_{i}} f(y, T) \mathrm{d} y .
$$

Therein, $a_{i}=\mathcal{L}\left(\mathcal{M}^{-1}\right)_{i i}, \quad i_{0}=N_{c} \mathcal{L I}_{0} / \phi_{0}$ and $\quad f(y, T)=\frac{1}{2}\left[g(y, T)+g\left(y+\frac{1}{2}, T\right)\right] / 2$. The zero-mean, rescaled noise is $\tilde{\Gamma}(s)=\sqrt{\tau_{0}} \Gamma\left(\tau_{0} s\right)$ yielding $\left\langle\tilde{\Gamma}_{i}\left(s_{1}\right) \tilde{\Gamma}_{k}\left(s_{2}\right)\right\rangle=\delta_{i k} \delta\left(s_{1}-s_{2}\right)$. Its intensity $D=k_{\mathrm{B}} T / 2 \varepsilon_{0}=\left(T / T^{*}\right) D^{*}$, where $\varepsilon_{0}=\phi_{0}^{2} / 2 \mathcal{L}$ is the magnetic energy and $D^{*}=k_{\mathrm{B}} T^{*} / 2 \varepsilon_{0}$ denotes the ratio of the characteristic thermal energy to the magnetic energy [4]. Both these characteristic energies are of quantum origin: $T^{*}$ is the temperature corresponding to the energy gap at the Fermi surface and $\varepsilon_{0}$ relates to the magnetic flux quantum $\phi_{0}$.

\section{Fokker-Planck equation}

Due to the symmetry $\mathcal{M}_{i k}=\mathcal{M}_{k i}$, the set of Eqs. (8) is a gradient system independent of the specific configuration of the cylinders. The case with $N=1$ has been studied previously in [4] for which the potential (9) is in general multi-stable. If the so-called flux trapping is absent, the potential (9), which is reflection invariant, can be either mono-stable or bistable, depending whether the temperature of the system is above or below a critical temperature $T_{c}$. The maxima of the corresponding probability density can be interpreted as self-sustaining fluxes (or currents) in the system. They are long living states, provided the time of thermal activation from one maximum to the other is huge in comparison with the decay time in the basin of attraction of a single maximum [4, 13]; it is in this sense, that the mean flux or current does vanish. The mean flux in a finite chain of mesoscopic cylinders also vanishes, due to the symmetry of the potential (9). A non-zero mean flux can occur only in the limit of infinitely many cylinders. We thus consider a system of coaxially stacked interacting cylinders in the limit $N \rightarrow \infty$. This scheme has successfully been used for investigating equilibrium, non-equilibrium and non-thermodynamic phase transitions, see e.g. $[14,15]$. The Fokker-Planck equation for the probability density $p\left(\left\{x_{i}\right\}, s\right)$ of the $N$-cylinder system in Eq. (8) reads [16]

$$
\frac{\partial}{\partial s} p\left(\left\{x_{i}\right\}, s\right)=\sum_{i=1}^{\infty} \frac{\partial}{\partial x_{i}}\left[V^{\prime}\left(x_{i}, T\right)+\sum_{j(\neq i)}^{N} \mathcal{L}\left(\mathcal{M}^{-1}\right)_{i j} x_{j}\right] p\left(\left\{x_{i}\right\}, s\right)+D \sum_{i} \frac{\partial^{2}}{\partial x_{i}^{2}} p\left(\left\{x_{i}\right\}, s\right) .
$$

Integration over all variables except $x_{k}$ yields the nonlinear, steady-state equation for the 1-dimensional probability density [17]

$$
\frac{\partial}{\partial x_{k}}\left[V^{\prime}\left(x_{k}, T\right)+\sum_{i \neq k}^{N} \mathcal{L}\left(\mathcal{M}^{-1}\right)_{i k}\left\langle x_{i} \mid x_{k}\right\rangle\right] p_{s}\left(x_{k}\right)+D \frac{\partial^{2}}{\partial x_{k}^{2}} p_{s}\left(x_{k}\right)=0,
$$


where $\left\langle x_{i} \mid x_{k}\right\rangle=\int x_{i} p_{s}\left(x_{i} \mid x_{k}\right) \mathrm{d} x_{i}$ is a stationary conditional expectation value of $x_{i}$ with respect to the conditional probability density $p_{s}\left(x_{i} \mid x_{k}\right)$. This equation is formally exact. It contains, however, the unknown quantity $\left\langle x_{i} \mid x_{k}\right\rangle$ which can be determined only via an approximation scheme. Thus, we invoke the following approximation: We rewrite the conditional expectation value as $\left\langle x_{i} \mid x_{k}\right\rangle=\left\langle x_{i}\right\rangle+c_{i k}$, wherein $c_{i k}=\left\langle x_{i} \mid x_{k}\right\rangle-\left\langle x_{i}\right\rangle$ accounts for correlations between $i$-th and $k$-th cylinder. In the limit, when $N \rightarrow \infty$, the system becomes statistically homogeneous so that the stationary average $\left\langle x_{k}\right\rangle=\langle x\rangle$ no longer depends on the index $k$. In this limit we shall neglect the correlations, i.e. we put $c_{i k}=0$. Following [17], we deduce that the stationary probability density for $x=x_{k}$ satisfies the non-linear FokkerPlanck equation [14],

$$
\frac{\mathrm{d}}{\mathrm{d} x}\left[V^{\prime}(x, T)-\lambda \mu\right] p_{s}(x)+D \frac{\mathrm{d}^{2}}{\mathrm{~d} x^{2}} p_{s}(x)=0,
$$

where

$$
\mu \equiv\langle x\rangle=\int_{-\infty}^{\infty} x p_{s}(x) \mathrm{d} x
$$

is the order parameter of the system and $\lambda=-\sum_{i \neq k} \mathcal{L}\left(\mathcal{M}^{-1}\right)_{i k}$ denotes an effective coupling constant. In this sum, the index $k$ is fixed and $i \in(-\infty, \infty)$. However, for the system of infinitely many cylinders, the result does not depend on $k$. Similarly, the parameter $a_{i}$ in the potential (9) does not depend in the index $i$, $a=a_{i}$ (below in all figures we take $a=1$ ). The mutual inductance for the coaxial alignment, which is expressed by a complex formula involving elliptic integrals, is positive [9], $M_{i k}>0$. The predominant non-diagonal elements $\left(\mathcal{M}^{-1}\right)_{i k}$ of the inverse matrix $\mathcal{M}^{-1}$ are negative and as a result the coupling constant is positive, i.e. $\lambda>0$. Its value is typically small and for generic cases $\lambda<0.1$. We expect, nevertheless a "ferromagnetic" state of the system, characterized by the parallel alignment of the magnetic moments induced by the currents flowing in the neighboring cylinders. Indeed, the solution of Eq. (12) reads

$$
p_{s}(x)=p_{s}(x, \mu)=N_{0}(\mu) \exp [-(V(x, T)-\lambda \mu x) / D],
$$

where $N_{0}(\mu)$ is the normalization constant and $D \equiv D(T) \propto T$, see below (9).

\section{State equation}

From (13) and (14) one finds the self-consistent steady-state equation

$$
\mu=F(\mu),
$$

where

$$
F(\mu)=\frac{\int_{-\infty}^{\infty} x \exp [-(V(x, T)-\lambda \mu x) / D(T)] \mathrm{d} x}{\int_{-\infty}^{\infty} \exp [-(V(x, T)-\lambda \mu x) / D(T)] \mathrm{d} x} .
$$

The closed form of this nonlinear equation allows one to study various regimes. The flux state is characterized by the non-vanishing mean flux $\mu=\langle x\rangle \neq 0$. If an external magnetic field is applied then trivially $\mu \neq 0$. The non-trivial case emerges when the external flux is zero but $\mu \neq 0$. Because the potential $V(x, T)=V(-x, T)$, the Eq. (15) has always the solution $\mu=0$. This solution becomes unstable, however, when the parameters exceed a critical value: a bifurcation into two stable states characterized by $\mu \neq 0$ is then expected. These states are symmetric with respect to the inversion of the current. The numerical solution of (15) depicted with Fig. 1 indeed confirms our expectation. Therein, we elucidate 


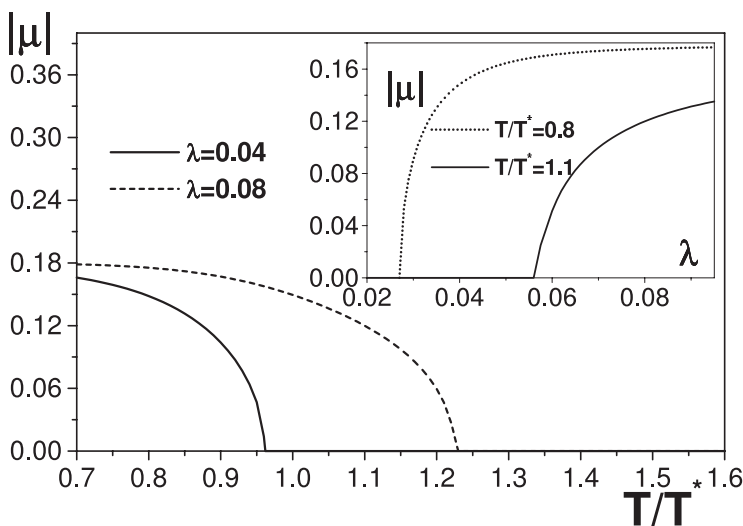

Fig. 1 Phase transition from disordered (zero flux) to ordered (finite flux) states in the coaxial system of coupled mesoscopic cylinders. The order parameter is the averaged magnetic flux $\mu$ through one cylinder. The corresponding state of the system is controlled by the dimensionless temperature $T / T^{*}$, or (see the inset) by the coupling constant $\lambda$.

both, the role of temperature and the interaction strength for two sets of parameters. The temperature affects the system via the 'single particle potential' $V(x, T)$ and the noise intensity $D$. The coupling constant $\lambda$ depends on the distance between cylinders. The dimensionless parameters of the model are chosen so as to yield $D=0.001 T / T^{*}$ and $i_{0}=1$ (the potential $V(x, T)$ then becomes bistable below $\left.T=1.66 T^{*}\right)$.

We note that the continuous phase transition does occur at the transition point determined by the relation

$$
\left.\frac{\mathrm{d} F(\mu)}{\mathrm{d} \mu}\right|_{\mu=0}=1 .
$$

The critical coupling constant $\lambda_{c}$ is consequently obtained as

$$
\lambda_{c}=\frac{D(T)}{\left\langle x^{2}\right\rangle_{0}}
$$

where

$$
\left\langle x^{n}\right\rangle_{0}=\frac{\int_{-\infty}^{\infty} x^{n} \exp [-V(x, T) / D(T)] \mathrm{d} x}{\int_{-\infty}^{\infty} \exp [-V(x, T) / D(T)] \mathrm{d} x} .
$$

The bifurcation diagram obtained from this equation is depicted with Fig. 2 . We find that in the vicinity of the transition point $\lambda_{c}$, the order parameter $|\mu|$ behaves algebraically; i.e.

$$
\mu^{2}=\frac{2}{K(T)}\left(\frac{\lambda_{c}}{\lambda}\right)^{2}\left[1-\frac{\lambda_{c}}{\lambda}\right], \quad \lambda>\lambda_{c},
$$

where the kurtosis

$$
K(T)=1-\frac{\left\langle x^{4}\right\rangle_{0}}{3\left\langle x^{2}\right\rangle_{0}^{2}}
$$

characterizes the deviation of the steady state of the non-interacting system from the Gaussian distribution. It is a monotonically decreasing function of temperature that starts from a positive value at $T=0$ and successively diminishes towards zero as $T$ increases (approaching the onset to the Gaussian regime). 


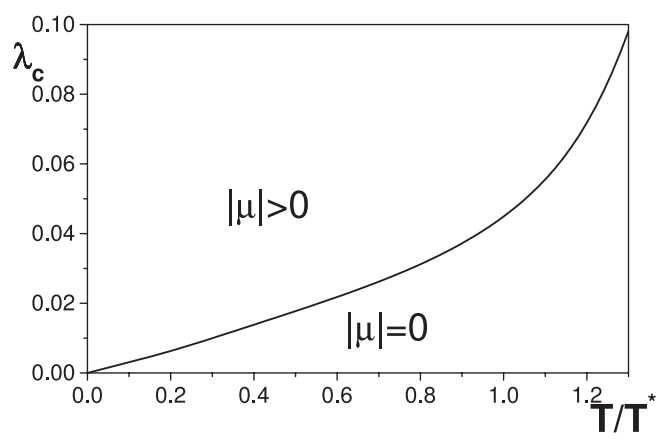

Fig. 2 Phase diagram for the chain of mesoscopic cylinders: the critical coupling constant $\lambda_{c} v s$. the reduced temperature. In the main panel the low temperature case is shown. The inset depicts the extended domain of bifurcation parameters with saturation occurring at strong coupling.

From (20) it follows that near the transition point $\mu \propto\left(\lambda-\lambda_{c}\right)^{\beta}$ for $\lambda>\lambda_{c}$, where the critical exponent $\beta=1 / 2$, i.e. it assumes the classical value.

\section{Summary}

The studied system of mesoscopic cylinders presents a 3-dimensional many-degree-of-freedom system. A related system has been studied in [18]. Here, we have reduced its description to the quasi 1-dimensional system modeled by a set of Langevin equations (8). The properties of magnetic flux depend on the mutual interplay of quantum coherence and dissipation. Classical dissipation at finite temperature is taken into account via Eq. (3) and quantum mechanically via Eq. (4). The experimental verification of this collective behavior can serve as an indirect evidence that the constituents, i.e. single cylinders, are monostable or bistable systems. A wire made of single wall carbon nanotubes (e.g. a long nanotube with periodically distributed defects like carbon peapods [19]) or coaxial and uniradial loop-by-loop winding of nanobelts [20] are proposed to be suitable test systems. Such systems have potential applications in investigating fundamental physical phenomena and could be used as "flux guides" or "magnetic fibres" in close analogy to wave guides and optical fibres in modern photonics.

In conclusion, we have shown that the co-operativity between mesoscopic-sized, coaxially coupled cylinders yields critical behavior and phase transitions from the zero mean to the non-zero mean flux state. A macroscopic stationary non-zero current can thus flow in absence of external driving.

Acknowledgements Financial support by the Ministry of Scientific Research and Information Technology via the grant No PBZ-MIN-008/P03/2003 (J. D. and M. Sz.), the ESF (Stochastic Dynamics: fundamentals and applications), the Foundation for Polish Science (P. H.) and DAAD-KBN (Stochastic Complexity) is gratefully acknowledged.

\section{References}

[1] Y. Imry, Introduction to Mesoscopic Physics (Oxford University Press, New York, 1997).

[2] D. Wohlleben, M. Esser, P. Freche, E. Zipper, and M. Szopa, Phys. Rev. Lett. 66, 3191 (1991).

M. Szopa, D. Wohlleben, and E. Zipper, Phys. Lett. A 160, 271 (1991).

M. Szopa and E. Zipper, Int. J. Mod. Phys. B 9, 161 (1995).

[3] M. Szopa, M. Margańska, and E. Zipper, Phys. Lett A 299, 593 (2002).

[4] J. Dajka, J. Łuczka, M. Szopa, and E. Zipper, Phys. Rev. B 67, 073305 (2003).

[5] F. Hund, Ann. Phys. (Leipzig) 32, 102 (1938).

U. Eckern and P. Schwab, J. Low Temp. Phys. 126, 1291 (2002).

[6] H. Bouchiat, in: Mesoscopic Quantum Physics, edited by E. Akkermans et al. (North-Holland, Amsterdam, 1995).

[7] Sh. Kogan, Electronic Noise and Fluctuations in Solids (Cambridge University Press, Cambridge, 1996).

[8] T. Dittrich, P. Hänggi, G.-L. Ingold, B. Kramer, G. Schön, and W. Zwerger, Quantum Transport and Dissipation (Wiley-VCH, Weinheim, 1998).

[9] J. X. Zhu, Z. D. Wang, and Q. Wang, J. Phys. Soc. Jpn. 65, 2602 (1996). 
[10] J. D. Jackson, Classical Electrodynamics (Wiley, New York, 1975).

[11] This relation is analogous to the one for the resistively shunted junction model, cf. chapter 9 and Eq. (9.1.3.1) in [7].

[12] R. Landauer and M. Buttiker, Phys. Rev. Lett. 54, 2049 (1985).

[13] P. Hänggi, P. Talkner, and M. Borkovec, Rev. Mod. Phys. 62, 251 (1990).

[14] A. Pikovsky, Lect. Notes Phys. 484, 210 (1997).

[15] M. Shiino, Phys. Rev. A 36, 2393 (1987).

K. Kometai and H. Shimizu, J. Stat. Phys. 13, 473 (1975).

R. C. Desai and R. Zwanzig, J. Stat. Phys. 19, 1 (1978).

D. A. Dawson, J. Stat. Phys. 31, 29 (1983).

[16] P. Hänggi and H. Thomas, Phys. Rep. 168, 207 (1982).

[17] C. Van den Broeck, J. M. R. Parrondo, and R. Toral, Phys. Rev. Lett. 73, 3395 (1994).

[18] M. Lisowski, E. Zipper, and M. Stebelski, Phys. Rev. B 59, 8305 (1999).

[19] B. W. Smith, M. Monthioux, and D. E. Luzzi, Nature 396, 323 (1998).

S. Iijima, Physica B 323, 1 (2002).

[20] X. Y. Kong, Y. Ding, R. Yang, and Z. L. Wang, Science 303, 1348 (2004). 